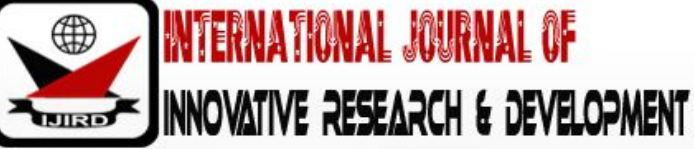

ISSN 2278 - 0211 (Online)

\section{Assessment of Students' Perception of Content Difficulty in the Nigerian Further Mathematics Curriculum}

\author{
Zalmon, Ibaan Gogo \\ Lecturer, Department of Mathematics/ Statistics, Faculty of Natural and Applied Sciences, \\ Ignatius Ajuru University of Education, Port Harcourt, Nigeria \\ George, Nchelem Rosemary \\ Senior Lecturer, Department of Mathematics/ Statistics, Faculty of Natural and Applied Sciences, \\ Ignatius Ajuru University of Education, Port Harcourt, Nigeria
}

\begin{abstract}
:
The study assessed the extent of students' perception of content difficulty in the Nigerian Further Mathematics Curriculum (FMC).The descriptive survey research design was adopted for the study. The study was conducted in Gokana local government area of Rivers State, Nigeria with a population of sixty (60) senior secondary class three students offering Further Mathematics from the twelve (12) public senior secondary schools in the area. Census sampling technique was used to select the sample of 60 students used for the study. The instrument for data collection was the researchers' made and validated Further Mathematics Curriculum Content Difficulty Assessment Questionnaire (FMCCDAQ). The test-retest reliability method and Pearson's Product Moment Correlation (PPMC) statistic were used to obtain 0.73 reliability coefficient for the FMCCDAQ. Six research questions guided the study. Mean, standard deviation and simple percentages were used for data analysis. The study found out that students perceived $88.20 \%$ of the Further Mathematics curriculum content difficult to learn with learning difficulties in all the FMC themes of pure mathematics, coordinate geometry, statistics, mechanics and operations research indicating poor FMC implementation. The study among others recommended a holistic review of the Nigerian FMC contents and improved instructional effectiveness through training and re-training of Further Mathematics teachers.
\end{abstract}

Keywords: Perception, content, difficulty, further mathematics, curriculum

\section{Introduction}

Mathematics education has been given a prime place at the basic and post basic levels of education in Nigeria because of its significant contributory role in the social, economic, scientific and technological development of the nation. The national policy on education specified the compulsory teaching and learning of Mathematics at the basic and post basic education levels (Federal Republic of Nigeria (FRN), 2014). The educational policy provided for students to offer the compulsory cross-cutting General Mathematics and the optional Further Mathematics recommended for students with high ability in General Mathematics who require a good foundation for future studies in Mathematics or Mathematics related courses at the senior secondary education level (FRN, 2014; Nigerian Educational Research and Development Council (NERDC), 2012).

The Further Mathematics Curriculum (FMC) was first designed in 1985 by the Federal Ministry of Education with three broad themes of Pure Mathematics, Mechanics and Statistics and three objectives (NERDC, 2012). The objectives of the FMC according to the NERDC (2012) are to: helpthe students to develop conceptual and manipulative skills in Mathematics so as to prepare them for further studies in Mathematics and its application; reflect continuity with those used in Universities, Polytechnics, Federal Colleges of Education and Colleges of Science and Technology, so that graduates of the curriculum have nothing to unlearn on entering any of the above mentioned institutions; prepare potential Mathematicians, Engineers and Scientists. The FMC was reviewed in 2007 by the Nigerian Educational Research and Development Council with the addition of Coordinate Geometry and Operations Research to the previous themes but retaining the same objectives. The five themes of the FMC are Pure Mathematics, Mechanics, Statistics, Coordinate Geometry and Operations Research. This curriculum review was necessitated by the nation's need to develop a new tradebased Mathematics curriculum designed to meet the targets of the National Economic Empowerment and Development Strategy (NEEDS) which emphasised value re-orientation, poverty eradication, job creation, wealth generation and citizenry empowerment through the instrument of education (NERDC, 2012).The implementation of this trade curriculum commenced in the year 2011(NERDC, 2012).

All school subjects have curriculum that guides its classroom content delivery and Further Mathematics is not an exemption. Further Mathematics curriculum is defined as the formal document which prescribes the content of advance mathematical concepts that the teacher is expected to implement in the classroom alongside with performance objectives, 
teacher and student activities, the instructional strategies and materials. The curriculum content of Further Mathematics can simply be referred to as advance Mathematics. The above definition implies that Further Mathematics can also be defined as higher Mathematics. This may suggest why some countries call it higher or advanced Mathematics. It also describes a course studied in addition to the General Mathematics. The study of Further Mathematics involves studying both pure and applied Mathematics. The pure part of Further Mathematics is of a higher standard than those of the General Mathematics. The topics covered in Further Mathematics are more sophisticated and conceptually advanced compared to the General Mathematics. Further Mathematics affords Senior Secondary School students' opportunity to be introduced to some topics in Advanced Level mathematics in order to prepare them to study mathematics or mathematics related courses in their next level of education (Charles-Ogan and George, 2019).

Further Mathematics is a highly respected subject and is recognized for its challenging content. It is recommended for students with high ability in General Mathematics who will need to acquire a foundation for future studies in Mathematics or the Mathematics related sciences. Despite the fact that the subject is recommended for high ability students, it does not rule out the fact that students who offer it are free of difficulties that may arise from its learning. To this end Iji and Omenka (2015) opined that some of the reasons that make students experience difficulties in Nigerian Mathematics programmes are non-preparedness of Mathematics teachers to handle the teaching of the curriculum contents, insufficient time to teach and overload of the curriculum. It is over a decade since the trade-based Mathematics curriculum was designed and implemented. With the introduction of new and advance curriculum contents in the FMC, the researchers deemed it fit to embark on this study, so as to ascertain the students' perception of content difficulty in the Nigerian Further Mathematics Curriculum.

\subsection{Statement of the Problem}

Research reports revealed abysmal performance of senior secondary students in General Mathematics in external examinations with students perceiving some contents of the General Mathematics Curriculum (GMC) difficult to learn (Zalmon \& Wonu, 2017; Zalmon \& George, 2018). If the same students offering General Mathematics and Further Mathematics had perceived learning difficulties in the GMC content which is a prerequisite to learning the FMC content, it is expected that their perception of content difficulty in the FMC will be higher with a corresponding poor performance in external examinations. Therefore, the investigation delved into finding answer to the question: what is the extent of students' perception of content difficulty in the Nigerian Further Mathematics curriculum?

\subsection{Aim and Objectives of the Study}

The aim of this study was to assess the extent of students' perception of content difficulty in the Nigerian Further Mathematics Curriculum (FMC).The objectives were to:

- Assess the extent of students' perception of the Pure Mathematics content difficulty in the FMC.

- Determine the extent of students' perception of the Coordinate Geometry content difficulty in the FMC.

- Ascertain the extent of students' perception of the Statistics content difficulty in the FMC.

- Find out the extent of students' perception of the Mechanics content difficulty in the FMC.

- Evaluate the extent of students' perception of the Operations Research content difficulty in the FMC.

- Assess the extent of student perception of the FMC content difficulty.

\subsection{Research Questions}

Six research questions guided the study:

- What is the extent of students' perception of the Pure Mathematics content difficulty in the FMC?

- What is the extent of students' perception of the Coordinate Geometry content difficulty in the FMC?

- What is the extent of students' perception of the Statistics content difficulty in the FMC?

- What is the extent of students' perception of the Mechanics content difficulty in the FMC?

- What is the extent of students' perception of the Operations Research content difficulty in the FMC?

- What is the extent of students' perception of the FMC content difficulty?

\section{Methodology}

The descriptive survey research design was adopted for the study. The study was conducted in Gokana local government area of Rivers State, Nigeria with a population of sixty (60) senior secondary class three students offering Further Mathematics from the twelve (12) public senior secondary schools in the area. The senior secondary class three students constituted the population of the study because the study was interested in assessing the perception of difficulty of students who had been taught most of the Further Mathematics curriculum contents. Further Mathematics is optional and few schools and students offer the subject. Census sampling technique was used to select all the 60 students in the population of the study used for the study as sample. The census sampling technique was deemed fit for this study because the elements of the population was small for selection to be carried out. The instrument for data collection was the researchers' made Further Mathematics Curriculum Content Difficulty Assessment Questionnaire (FMCCDAQ). The FMCCDAQ consisted of 263FMC contents patterned after the four-point Likert rating scale of Very Difficult (VD) - 4 points, Difficult (D) - 3 points, Easy (E) - 2 points and Very Easy (VE) - 1 point with a criterion mean of 2.50.The decision rule was: Difficult content (mean $\geq 2.50$ ); Easy content (mean<2.50). The FMCCDAQ had two sections. Section A and section B. Section A was used to elicit demographic information such as class and gender from the respondents while section B was used to obtain the response of the respondents on their perception of the Further Mathematics curriculum content 
difficulty based on the five themes of the curriculum namely; Pure Mathematics, Coordinate Geometry, Statistics, Mechanics and Operations Research. Three experts in Curriculum Studies and Mathematics Education validated the instrument face and content wise. The test-retest reliability method and Pearson's Product Moment Correlation (PPMC) statistic were used to obtain 0.73 reliability coefficient for FMCCDAQ. The FMCCDAQ was administered to the participants on a face to face mode by the researchers with the assistant of theFurther Mathematics teachers. Mean, standard deviation and simple percentages were the statistical tools used for data analysis. The decisionrule was: Difficult content $($ mean $\geq 2.50)$; Easy content $($ mean $<2.50)$.

\section{Results}

- Research question one: What is the extent of students' perception of the Pure Mathematics content difficulty in the FMC?

\begin{tabular}{|c|c|c|c|c|c|c|c|c|}
\hline $\mathbf{S} / \mathbf{N}$ & Pure Mathematics Contents & VD & $\mathbf{D}$ & $\mathbf{E}$ & VE & Mean & SD & Decision \\
\hline & Definition of sets & 0 & 6 & 23 & 31 & 1.58 & 0.67 & E \\
\hline & Set notation methods & 14 & 13 & 15 & 18 & 2.38 & 1.15 & E \\
\hline & Null set & 16 & 15 & 13 & 16 & 2.52 & 1.16 & $\bar{D}$ \\
\hline & Singleton set & 12 & 20 & 11 & 17 & 2.45 & 1.11 & $\mathrm{E}$ \\
\hline & Finite and infinite set & 2 & 14 & 25 & 19 & 1.98 & 0.83 & $\mathrm{E}$ \\
\hline & Subsets & 5 & 11 & 14 & 30 & 1.85 & 1.01 & $\mathrm{E}$ \\
\hline & Universal set & 0 & 8 & 14 & 38 & 1.50 & 0.72 & $\mathrm{E}$ \\
\hline & Power set & 3 & 10 & 28 & 19 & 1.95 & 0.83 & $\mathrm{E}$ \\
\hline & Union of sets & 0 & 11 & 20 & 29 & 1.70 & 0.77 & $\mathrm{E}$ \\
\hline & Intersection of set & 2 & 2 & 27 & 29 & 1.62 & 0.72 & $\mathrm{E}$ \\
\hline & Complements of set & 5 & 2 & 35 & 18 & 1.90 & 0.82 & E \\
\hline & Number of elements in a set & 7 & 14 & 22 & 17 & 2.18 & 0.98 & E \\
\hline & $\begin{array}{l}\text { Venn diagram and applications up to } 3 \text { set } \\
\text { problem }\end{array}$ & 10 & 16 & 19 & 15 & 2.35 & 1.04 & E \\
\hline & Definition of binary operation & 8 & 9 & 23 & 20 & 2.08 & 1.01 & $\mathrm{E}$ \\
\hline & Association law of binary operation & 13 & 21 & 13 & 13 & 2.57 & 1.06 & $\mathrm{D}$ \\
\hline & Commutative law of binary operation & 13 & 17 & 16 & 14 & 2.48 & 1.08 & $\mathrm{E}$ \\
\hline & Distributive law of binary operation & 19 & 11 & 17 & 13 & 2.60 & 1.15 & $\mathrm{D}$ \\
\hline & Laws of complementation as insets & 22 & 18 & 15 & 5 & 2.95 & 0.98 & $\mathrm{D}$ \\
\hline & Identify elements & 14 & 22 & 19 & 5 & 2.75 & 0.91 & $\mathrm{D}$ \\
\hline & Inverse of an element & 22 & 19 & 17 & 2 & 3.02 & 0.89 & $\mathrm{D}$ \\
\hline & Multiplication tables of binary operation & 17 & 6 & 26 & 11 & 2.48 & 1.10 & $\mathrm{E}$ \\
\hline & Definition of indices & 9 & 5 & 23 & 23 & 2.00 & 1.04 & $\mathrm{E}$ \\
\hline & Multiplicative laws of indices & 9 & 3 & 30 & 18 & 2.05 & 0.98 & $\mathrm{E}$ \\
\hline & Divisional law of indices & 14 & 2 & 31 & 13 & 2.28 & 1.06 & $\mathrm{E}$ \\
\hline & Power law of indices & 14 & 4 & 25 & 17 & 2.25 & 1.11 & $\mathrm{E}$ \\
\hline & Zero power law of indices & 9 & 13 & 19 & 19 & 2.20 & 1.05 & $\mathrm{E}$ \\
\hline & Negative power law of indices & 13 & 13 & 15 & 19 & 2.33 & 1.14 & $\mathrm{E}$ \\
\hline & Inverse power law of indices & 8 & 23 & 14 & 15 & 2.40 & 1.01 & E \\
\hline & $\begin{array}{l}\text { Applications of indices, solution of indicial } \\
\text { equations up to quadratic equation }\end{array}$ & 28 & 13 & 7 & 12 & 2.95 & 1.19 & $\mathrm{D}$ \\
\hline & Logarithms & 2 & 2 & 36 & 20 & 1.77 & 0.67 & $\mathrm{E}$ \\
\hline & Definition of logarithm & 2 & 1 & 34 & 23 & 1.70 & 0.67 & $\mathrm{E}$ \\
\hline & Multiplicative laws of logarithm & 2 & 16 & 32 & 10 & 2.17 & 0.74 & $\mathrm{E}$ \\
\hline & Divisional law of logarithm & 9 & 10 & 33 & 8 & 2.33 & 0.90 & E \\
\hline & Power law of logarithm & 7 & 19 & 23 & 11 & 2.37 & 0.92 & E \\
\hline & Logarithm of number in the same base & 13 & 15 & 23 & 9 & 2.53 & 1.00 & $\mathrm{D}$ \\
\hline & Logarithm of number equal to 1 & 14 & 24 & 19 & 3 & 2.82 & 0.85 & $\mathrm{D}$ \\
\hline & Logarithm of a number equal to zero & 12 & 28 & 15 & 5 & 2.78 & 0.87 & $\mathrm{D}$ \\
\hline & Change of base of logarithm & 16 & 10 & 24 & 10 & 2.53 & 1.07 & $\mathrm{D}$ \\
\hline & Definition of surds & 10 & 10 & 32 & 8 & 2.37 & 0.92 & $\mathrm{E}$ \\
\hline & Rules for manipulating surds $(\sqrt{ } \mathrm{ab})$ & 23 & 21 & 12 & 4 & 3.05 & 0.93 & $\mathrm{D}$ \\
\hline & Multiplicative rule of surds $(\sqrt{ } \mathrm{ab})$ & 25 & 12 & 21 & 2 & 3.00 & 0.96 & $\mathrm{D}$ \\
\hline & Divisional rule of surds $(\sqrt{a b})$ & 29 & 13 & 16 & 2 & 3.15 & 0.94 & $\mathrm{D}$ \\
\hline & Power rule of surds $(\sqrt{a b})$ & 20 & 14 & 23 & 3 & 2.85 & 0.95 & $\mathrm{D}$ \\
\hline
\end{tabular}




\begin{tabular}{|c|c|c|c|c|c|c|c|c|}
\hline $\mathbf{S} / \mathbf{N}$ & Pure Mathematics Contents & VD & $\mathbf{D}$ & $\mathbf{E}$ & VE & Mean & SD & Decision \\
\hline & Inverse power rule of surd & 31 & 17 & 7 & 5 & 3.23 & 0.96 & $\mathrm{D}$ \\
\hline & Rationalization of the denominator & 34 & 12 & 9 & 5 & 3.25 & 1.00 & $\mathrm{D}$ \\
\hline & Definition of function & 32 & 14 & 9 & 5 & 3.22 & 0.99 & $\mathrm{D}$ \\
\hline & One to one function & 26 & 16 & 11 & 7 & 3.02 & 1.05 & $\mathrm{D}$ \\
\hline & Onto function & 27 & 21 & 6 & 6 & 3.15 & 0.97 & $\mathrm{D}$ \\
\hline & Inverse function & 24 & 25 & 4 & 7 & 3.10 & 0.97 & $\mathrm{D}$ \\
\hline & Identify function & 26 & 22 & 8 & 4 & 3.17 & 0.91 & $\mathrm{D}$ \\
\hline & Constant function & 26 & 13 & 15 & 6 & 2.98 & 1.05 & $\mathrm{D}$ \\
\hline & Circular function & 26 & 19 & 11 & 4 & 3.12 & 0.94 & $\mathrm{D}$ \\
\hline & Logarithmic function & 12 & 24 & 17 & 7 & 2.68 & 0.93 & $\mathrm{D}$ \\
\hline & Experiential function & 20 & 15 & 18 & 7 & 2.80 & 1.04 & $\mathrm{D}$ \\
\hline & Composite function & 26 & 21 & 8 & 5 & 3.13 & 0.95 & $\mathrm{D}$ \\
\hline & Application of functions & 26 & 22 & 5 & 7 & 3.12 & 0.99 & $\mathrm{D}$ \\
\hline & Solutions of problems of function & 26 & 20 & 9 & 5 & 3.12 & 0.96 & $\mathrm{D}$ \\
\hline & Definition of sequence & 12 & 15 & 20 & 13 & 2.43 & 1.05 & $\mathrm{E}$ \\
\hline & The nth them of a sequence & 10 & 9 & 20 & 21 & 2.13 & 1.08 & E \\
\hline & Definition of series & 16 & 6 & 25 & 13 & 2.42 & 1.11 & $\mathrm{E}$ \\
\hline & The nth term of a series & 13 & 10 & 19 & 18 & 2.30 & 1.12 & $\mathrm{E}$ \\
\hline & Arithmetic and geometric progressive & 18 & 11 & 13 & 18 & 2.48 & 1.21 & $\mathrm{E}$ \\
\hline & Linear inequalities in one variable & 21 & 10 & 16 & 13 & 2.65 & 1.18 & $\mathrm{D}$ \\
\hline & Linear inequalities in two variables & 24 & 8 & 17 & 11 & 2.75 & 1.17 & $\mathrm{D}$ \\
\hline & Graphs of linear inequalities in two variables & 28 & 12 & 17 & 3 & 3.08 & 0.98 & $\mathrm{D}$ \\
\hline & Quadratic inequalities & 15 & 26 & 18 & 1 & 2.92 & 0.79 & $\mathrm{D}$ \\
\hline & Inequalities in two dimensions & 25 & 20 & 14 & 1 & 3.15 & 0.84 & $\mathrm{D}$ \\
\hline & Calculating devices & 26 & 22 & 11 & 1 & 3.22 & 0.80 & $\mathrm{D}$ \\
\hline & Abacus calculating devices & 20 & 21 & 11 & 8 & 2.88 & 1.03 & $\mathrm{D}$ \\
\hline & Decimal system & 5 & 22 & 26 & 7 & 2.42 & 0.81 & $\mathrm{E}$ \\
\hline & Binary system & 16 & 1 & 38 & 5 & 2.47 & 0.98 & $\mathrm{E}$ \\
\hline & Flow charts & 33 & 10 & 14 & 3 & 3.22 & 0.98 & $\mathrm{D}$ \\
\hline & Application of flow charts & 31 & 11 & 15 & 3 & 3.17 & 0.98 & $\mathrm{D}$ \\
\hline & Trigonometric ratios of $30^{\circ}, 45^{0}, 60^{0}$ & 31 & 11 & 15 & 3 & 3.17 & 0.98 & $\mathrm{D}$ \\
\hline & $\begin{array}{l}\text { Application of trigonometric ratio of } 30^{\circ}, 45^{\circ} \text {, } \\
60^{\circ}\end{array}$ & 38 & 11 & 8 & 3 & 3.40 & 0.91 & $\mathrm{D}$ \\
\hline & $\begin{array}{c}\text { Six trigonometric functions of angles of any } \\
\text { magnitude (sine, cosine, tangent secant } \\
\text { cosecant cotangent) }\end{array}$ & 33 & 8 & 15 & 4 & 3.17 & 1.03 & $\mathrm{D}$ \\
\hline & Range or specified trigonometry & 30 & 19 & 10 & 1 & 3.30 & 0.81 & $\mathrm{D}$ \\
\hline & Domain of specified trigonometry & 30 & 12 & 17 & 1 & 3.18 & 0.91 & $\mathrm{D}$ \\
\hline & $\begin{array}{c}\text { Graphs of trigonometric ratios with emphasis } \\
\text { on their amplitude and periodicity }\end{array}$ & 30 & 18 & 9 & 3 & 3.25 & 0.89 & $\mathrm{D}$ \\
\hline & $\begin{array}{l}\text { Relationship between graphs of trigonometric } \\
\text { ratios }(\mathrm{y}=\mathrm{a} \sin (\mathrm{bx})+\mathrm{c}, \mathrm{y}=\mathrm{a} \cos (\mathrm{bx})+\mathrm{c}, \mathrm{y}=\mathrm{a}+ \\
\tan (\mathrm{bx})+\mathrm{c}\end{array}$ & 37 & 11 & 11 & 1 & 3.40 & 0.85 & $\mathrm{D}$ \\
\hline & Graphs of inverse by ratios & 33 & 11 & 15 & 1 & 3.27 & 0.90 & $\mathrm{D}$ \\
\hline & $\begin{array}{l}\text { Solutions of simple equation involving the six } \\
\text { trigonometric function }\end{array}$ & 25 & 18 & 9 & 8 & 3.00 & 1.06 & $\mathrm{D}$ \\
\hline & $\begin{array}{l}\text { Proofs of simple trigonometric identities }\left(\sin ^{2} \mathrm{x}\right. \\
+\cos ^{2} \mathrm{x}=1, \sec ^{2} \mathrm{x}=1+\tan ^{2} \mathrm{x}\end{array}$ & 28 & 18 & 4 & 10 & 3.07 & 1.10 & $\mathrm{D}$ \\
\hline & Sum of roots of quadratic equation $(\alpha+\beta=-b / a)$ & 17 & 19 & 14 & 10 & 2.72 & 1.06 & $\mathrm{D}$ \\
\hline & $\begin{array}{l}\text { Product of roots of quadratic equation }(\alpha \beta= \\
\qquad / a)\end{array}$ & 11 & 16 & 27 & 6 & 2.53 & 0.91 & $\mathrm{D}$ \\
\hline & $\begin{array}{c}\text { Finding quadratic equation given sum and } \\
\text { products of roots ( } \mathrm{x}^{2} \text { ( (sum of roots) + product) } \\
=0\end{array}$ & 14 & 16 & 24 & 6 & 2.63 & 0.96 & $\mathrm{D}$ \\
\hline & $\begin{array}{c}\text { Condition for quadratic equation to have equal } \\
\text { roots }\left(\mathrm{b}^{2}=4 \mathrm{ac}\right)\end{array}$ & 31 & 12 & 11 & 6 & 3.13 & 1.05 & $\mathrm{D}$ \\
\hline & $\begin{array}{l}\text { Condition for quadratic equation to have real } \\
\text { roots }\left(\mathrm{b}^{2}>4 \mathrm{ac}\right)\end{array}$ & 35 & 6 & 14 & 5 & 3.18 & 1.07 & $\mathrm{D}$ \\
\hline
\end{tabular}




\begin{tabular}{|c|c|c|c|c|c|c|c|c|}
\hline $\mathbf{S} / \mathbf{N}$ & Pure Mathematics Contents & VD & $\mathbf{D}$ & $\mathbf{E}$ & VE & Mean & SD & Decision \\
\hline & $\begin{array}{c}\text { Condition for quadratic equation to have no } \\
\text { roots }\left(\mathrm{b}^{2}<4 \mathrm{ac}\right)\end{array}$ & 27 & 12 & 16 & 5 & 3.02 & 1.03 & $\mathrm{D}$ \\
\hline & Condition for given line to intersect a curve & 24 & 15 & 14 & 7 & 2.93 & 1.06 & $\mathrm{D}$ \\
\hline & Condition for given line to be tangent to curve & 18 & 24 & 12 & 6 & 2.90 & 0.95 & $\mathrm{D}$ \\
\hline & Condition for given line not to intersect a curve & 20 & 17 & 17 & 6 & 2.85 & 1.01 & $\mathrm{D}$ \\
\hline & $\begin{array}{c}\text { Solution of problems on roots quadratic } \\
\text { equation }\end{array}$ & 25 & 9 & 20 & 6 & 2.88 & 1.08 & $\mathrm{D}$ \\
\hline & Definition of polynomials & 24 & 6 & 22 & 8 & 2.77 & 1.13 & $\mathrm{D}$ \\
\hline & $\begin{array}{l}\text { Division of polynomials by a polynomial of } \\
\text { lesser degree }\end{array}$ & 32 & 13 & 8 & 7 & 3.17 & 1.06 & $\mathrm{D}$ \\
\hline & Remainder theorem & 33 & 8 & 15 & 4 & 3.17 & 1.03 & $\mathrm{D}$ \\
\hline & Factorization of polynomial & 25 & 16 & 9 & 10 & 2.93 & 1.12 & $\mathrm{D}$ \\
\hline & Roots of cubic equation & 30 & 10 & 17 & 3 & 3.12 & 0.99 & $\mathrm{D}$ \\
\hline & Sum of roots & 17 & 18 & 22 & 3 & 2.82 & 0.91 & $\mathrm{D}$ \\
\hline & Product of roots & 14 & 18 & 25 & 3 & 2.72 & 0.88 & $\mathrm{D}$ \\
\hline & Sum of products of two roots & 19 & 16 & 22 & 3 & 2.85 & 0.94 & $\mathrm{D}$ \\
\hline & Logical reasoning & 20 & 24 & 10 & 6 & 2.97 & 0.96 & $\mathrm{D}$ \\
\hline & Definition of statement & 18 & 19 & 13 & 10 & 2.75 & 1.07 & $\mathrm{D}$ \\
\hline & Negation of statement & 15 & 27 & 11 & 7 & 2.83 & 0.94 & $\mathrm{D}$ \\
\hline & Contra-positive of statement & 19 & 23 & 9 & 9 & 2.87 & 1.03 & $\mathrm{D}$ \\
\hline & Antecedents and consequence of statement & 30 & 9 & 16 & 5 & 3.07 & 1.06 & $\mathrm{D}$ \\
\hline & Conditional statement & 29 & 8 & 15 & 8 & 2.97 & 1.13 & $\mathrm{D}$ \\
\hline & Fundamental issues in intelligent system & 32 & 12 & 13 & 3 & 3.22 & 0.96 & $\mathrm{D}$ \\
\hline & Fundamental definition & 30 & 11 & 15 & 4 & 3.12 & 1.01 & $\mathrm{D}$ \\
\hline & Modeling the world & 35 & 1 & 20 & 4 & 3.12 & 1.09 & $\mathrm{D}$ \\
\hline & $\begin{array}{c}\text { Introduction to propositional and predicate } \\
\text { logical resolution }\end{array}$ & 41 & 6 & 10 & 3 & 3.42 & 0.94 & $\mathrm{D}$ \\
\hline & Introduction to theorem proving & 37 & 8 & 9 & 6 & 3.27 & 1.06 & $\mathrm{D}$ \\
\hline & Pascal triangle & 37 & 6 & 10 & 7 & 3.22 & 1.11 & $\mathrm{D}$ \\
\hline & $\begin{array}{l}\text { Binomial expansion of }(\mathrm{a}+\mathrm{b})^{\mathrm{n}} \text { where } \mathrm{n} \text { is the } \\
\text { positive integer }\end{array}$ & 30 & 13 & 14 & 3 & 3.17 & 0.96 & $\mathrm{D}$ \\
\hline & $\begin{array}{c}\text { Binomial expansion of }(\mathrm{a}+\mathrm{b})-\mathrm{n} \text { where } \mathrm{n} \text { is the } \\
\text { negative integer }\end{array}$ & 28 & 16 & 11 & 5 & 3.12 & 0.99 & $\mathrm{D}$ \\
\hline & $\begin{array}{l}\text { Binomial expansion of }(\mathrm{a}+\mathrm{b})^{1 / \mathrm{n}} \text { where } 1 / \mathrm{n} \text { is the } \\
\text { fractional value }\end{array}$ & 30 & 22 & 5 & 3 & 3.32 & 0.83 & $\mathrm{D}$ \\
\hline & Finding the nth term & 24 & 7 & 26 & 3 & 2.87 & 1.02 & $\mathrm{D}$ \\
\hline & Application of binomial expansion & 31 & 9 & 19 & 1 & 3.17 & 0.94 & $\mathrm{D}$ \\
\hline & Limits of a function & 36 & 7 & 13 & 4 & 3.25 & 1.02 & $\mathrm{D}$ \\
\hline & Differentiation of polynomial & 37 & 6 & 16 & 1 & 3.32 & 0.93 & $\mathrm{D}$ \\
\hline & $\begin{array}{l}\text { Differentiation of transcendental functions such } \\
\text { as } \sin x, e^{a x}, \log 3 x\end{array}$ & 32 & 15 & 10 & 3 & 3.27 & 0.92 & $\mathrm{D}$ \\
\hline & Product rule of differentiation & 33 & 11 & 13 & 3 & 3.23 & 0.96 & $\bar{D}$ \\
\hline & Quotient rule of differentiation & 26 & 14 & 15 & 5 & 3.02 & 1.02 & $\mathrm{D}$ \\
\hline & Function of function (chain rule) & 23 & 17 & 14 & 6 & 2.95 & 1.02 & $\mathrm{D}$ \\
\hline & Application of differentiation to rate of change & 29 & 18 & 5 & 8 & 3.13 & 1.05 & $\mathrm{D}$ \\
\hline & Application of differentiation to gradient & 26 & 16 & 12 & 6 & 3.03 & 1.02 & $\mathrm{D}$ \\
\hline & $\begin{array}{l}\text { Application of differentiation to maximum and } \\
\text { minimum values }\end{array}$ & 28 & 15 & 12 & 5 & 3.10 & 1.00 & $\mathrm{D}$ \\
\hline & $\begin{array}{l}\text { Application of differentiation to equation of } \\
\text { motion }\end{array}$ & 26 & 20 & 9 & 5 & 3.12 & 0.96 & $\mathrm{D}$ \\
\hline & Higher derivative & 33 & 14 & 8 & 5 & 3.25 & 0.99 & $\mathrm{D}$ \\
\hline & Differentiation implicit function & 38 & 12 & 7 & 3 & 3.42 & 0.89 & $\mathrm{D}$ \\
\hline & Matrices as linear transformations & 36 & 14 & 7 & 3 & 3.38 & 0.88 & $\mathrm{D}$ \\
\hline & Determinants & 35 & 12 & 10 & 3 & 3.32 & 0.93 & $\mathrm{D}$ \\
\hline & Solutions of 2 and 3 simultaneous equations & 20 & 10 & 27 & 3 & 2.78 & 0.98 & $\mathrm{D}$ \\
\hline & $\begin{array}{l}\text { Proper rational functions with denominators as } \\
\text { linear factors (distinct and repeated) and others }\end{array}$ & 33 & 13 & 11 & 3 & 3.27 & 0.94 & $\mathrm{D}$ \\
\hline
\end{tabular}




\begin{tabular}{|c|c|c|c|c|c|c|c|c|}
\hline S/ N & Pure Mathematics Contents & VD & D & E & VE & Mean & SD & Decision \\
\hline & $\begin{array}{c}\text { Understand integration as the reverse process } \\
\text { of differentiation }\end{array}$ & 38 & 14 & 4 & 4 & 3.43 & 0.89 & $\mathrm{D}$ \\
\hline & $\begin{array}{c}\text { Integration of algebraic polynomials including } \\
\text { 1/x, logarithmic functions }\end{array}$ & 34 & 18 & 5 & 3 & 3.38 & 0.85 & $\mathrm{D}$ \\
\hline $\begin{array}{c}\text { Definite integrals and application to kinematics } \\
\text { apply to v-t and s-t graphs }\end{array}$ & 38 & 13 & 8 & 1 & 3.47 & 0.79 & $\mathrm{D}$ \\
\hline & Areas under the curve & 30 & 13 & 13 & 4 & 3.15 & 0.99 & $\mathrm{D}$ \\
\hline & Trapezoidal rule & 31 & 13 & 11 & 5 & 3.17 & 1.01 & $\mathrm{D}$ \\
\hline & Volume of solids of revolution & 29 & 17 & 10 & 4 & 3.18 & 0.95 & $\mathrm{D}$ \\
\hline
\end{tabular}

Table 1: Meanand Standard Deviation on the Extent of Students' Perception of the

Pure Mathematics Content Difficulty in the FMC Difficult (D) ; Easy (E), D=75\% ; E=25\%

Data in table 1 revealed that students perceived the Pure Mathematics contents of the FMC difficult to learn (Mean=2.82; $\mathrm{SD}=0.98)$. Table 1 also showed that the extent of students' perception of the Pure Mathematics content difficulty in the FMCwas high (75\%).

- Research question two: What is the extent of students' perception of the Coordinate Geometry content difficulty in the FMC?

\begin{tabular}{|l|c|c|c|c|c|c|c|c|}
\hline S/ N & Coordinate Geometry Contents & VD & $\mathbf{D}$ & $\mathbf{E}$ & $\mathbf{V E}$ & Mean & SD & Decision \\
\hline 1. & Gradient of a straight line & 30 & 10 & 12 & 8 & 3.03 & 1.12 & $\mathrm{D}$ \\
\hline 2. & Distance between two points & 25 & 8 & 20 & 7 & 2.85 & 1.10 & $\mathrm{D}$ \\
\hline 3. & Condition for parallelism & 31 & 11 & 13 & 5 & 3.13 & 1.03 & $\mathrm{D}$ \\
\hline 4. & Condition for perpendicularity & 24 & 20 & 12 & 4 & 3.07 & 0.94 & $\mathrm{D}$ \\
\hline 5. & Equation of a line & 26 & 15 & 15 & 4 & 3.05 & 0.98 & $\mathrm{D}$ \\
\hline 6. & Transform relationship into linear form & 24 & 17 & 12 & 7 & 2.97 & 1.04 & $\mathrm{D}$ \\
\hline 7. & Areas of triangles and quadrilateral & 24 & 24 & 8 & 4 & 3.13 & 0.89 & $\mathrm{D}$ \\
\hline 8. & Definition of circle & 19 & 8 & 26 & 7 & 2.65 & 1.05 & $\mathrm{D}$ \\
\hline 9. & Equation of circle given center and radius & 23 & 12 & 21 & 4 & 2.90 & 1.00 & $\mathrm{D}$ \\
\hline 10. & General equation of a circle & 26 & 12 & 16 & 6 & 2.97 & 1.06 & $\mathrm{D}$ \\
\hline 11. & Finding center and radius of a given circle & 25 & 11 & 15 & 9 & 2.87 & 1.13 & $\mathrm{D}$ \\
\hline 12. & $\begin{array}{c}\text { Finding equation of a circle given the end } \\
\text { point of the diameter }\end{array}$ & 29 & 13 & 12 & 6 & 3.08 & 1.05 & $\mathrm{D}$ \\
\hline 13. & Equation of circle passing through 3 points & 42 & 6 & 6 & 6 & 3.40 & 1.03 & $\mathrm{D}$ \\
\hline 14. & Equation of tangent to a circle & 28 & 17 & 9 & 6 & 3.12 & 1.01 & $\mathrm{D}$ \\
\hline 15. & Length of tangent to a circle & 30 & 21 & 6 & 3 & 3.30 & 0.85 & $\mathrm{D}$ \\
\hline 16. & Equation of parabola in rectangular Cartesian & 25 & 27 & 5 & 3 & 3.23 & 0.81 & $\mathrm{D}$ \\
& coordinate & & & & & & & \\
\hline 17. & Equation of ellipse in rectangular Cartesian \\
& coordinate & 25 & 28 & 4 & 3 & 3.25 & 0.79 & $\mathrm{D}$ \\
\hline 18. & Parametric equation & 33 & 19 & 5 & 3 & 3.37 & 0.84 & $\mathrm{D}$ \\
\hline & Grand Mean & & & & & 3.08 & 0.99 & $\mathrm{D}$ \\
\hline
\end{tabular}

Table 2: Mean And Standard Deviation (SD) On the Extent of Students' Perception of the Coordinate Geometry Content Difficulty in the FMC Difficult (D); Easy (E), D=100\%; E=0\%

Data in table 2 revealed that students perceived the Coordinate Geometry content of the FMC difficult to learn (Mean=3.08; $\mathrm{SD}=0.99$ ). Table 2 also showed that the extent of student perception of the Coordinate Geometry content difficulty in the FMCwas high (100\%).

- $\quad$ Research question three: What is the extent of students' perception of the Statistics content difficulty in the FMC? 


\begin{tabular}{|c|c|c|c|c|c|c|c|c|}
\hline $\mathbf{S} / \mathbf{N}$ & Statistics Contents & VD & $\mathbf{D}$ & $\mathbf{E}$ & VE & Mean & SD & Decision \\
\hline 1. & Mean & 10 & 11 & 24 & 15 & 2.27 & 1.02 & $\mathrm{E}$ \\
\hline 2. & Mode & 8 & 7 & 29 & 16 & 2.12 & 0.96 & $\mathrm{E}$ \\
\hline 3. & Median & 9 & 15 & 22 & 14 & 2.32 & 1.00 & E \\
\hline 4. & Deciles & 18 & 20 & 11 & 11 & 2.75 & 1.08 & $\mathrm{D}$ \\
\hline 5. & Percentile & 19 & 20 & 9 & 12 & 2.77 & 1.11 & $\mathrm{D}$ \\
\hline 6. & Quartiles & 13 & 18 & 17 & 12 & 2.53 & 1.05 & $\mathrm{D}$ \\
\hline 7. & Range & 5 & 19 & 23 & 13 & 2.27 & 0.90 & $\mathrm{D}$ \\
\hline 8. & Inter-quartiles & 18 & 23 & 14 & 5 & 2.90 & 0.93 & $\mathrm{D}$ \\
\hline 9. & Mean deviation & 11 & 17 & 19 & 13 & 2.43 & 1.03 & $\mathrm{E}$ \\
\hline 10. & Standard deviation & 11 & 12 & 30 & 7 & 2.45 & 0.93 & $E$ \\
\hline 11. & Coefficient of variation & 14 & 15 & 21 & 10 & 2.55 & 1.03 & $\mathrm{D}$ \\
\hline 12. & Classical & 35 & 14 & 8 & 3 & 3.35 & 0.90 & $\mathrm{D}$ \\
\hline 13. & Frequential & 32 & 15 & 9 & 4 & 3.25 & 0.95 & $\mathrm{D}$ \\
\hline 14. & Axiomative approaches to probability & 33 & 17 & 6 & 4 & 3.32 & 0.91 & $\mathrm{D}$ \\
\hline 15. & Sample space & 37 & 9 & 11 & 3 & 3.33 & 0.95 & $\mathrm{D}$ \\
\hline 16. & Event space & 30 & 12 & 15 & 3 & 3.15 & 0.97 & $\mathrm{D}$ \\
\hline 17. & Mutually exclusive event & 37 & 8 & 12 & 3 & 3.32 & 0.97 & $\mathrm{D}$ \\
\hline 18. & Independent event & 32 & 13 & 10 & 5 & 3.20 & 1.01 & $\mathrm{D}$ \\
\hline 19. & Conditional event & 39 & 8 & 10 & 3 & 3.38 & 0.94 & $\mathrm{D}$ \\
\hline 20. & Conditional probability & 33 & 6 & 14 & 7 & 3.08 & 1.12 & $\mathrm{D}$ \\
\hline 21. & Probability trees & 30 & 8 & 14 & 8 & 3.00 & 1.13 & $\mathrm{D}$ \\
\hline 22. & Permutation on arrangement & 25 & 14 & 10 & 11 & 2.88 & 1.15 & $\mathrm{D}$ \\
\hline 23. & Cyclic permutation & 31 & 6 & 16 & 7 & 3.02 & 1.13 & $\mathrm{D}$ \\
\hline 24. & Arrangement of identical objects & 27 & 15 & 8 & 10 & 2.98 & 1.13 & $\mathrm{D}$ \\
\hline 25. & Arrangement in which repetitions are allowed & 33 & 10 & 6 & 11 & 3.08 & 1.18 & $\mathrm{D}$ \\
\hline 26. & Introduction to combination on selection & 30 & 19 & 5 & 6 & 3.22 & 0.98 & $\mathrm{D}$ \\
\hline 27. & Conditional arrangements and selection & 26 & 21 & 7 & 6 & 3.12 & 0.98 & $\mathrm{D}$ \\
\hline 28. & $\begin{array}{l}\text { Probability arrangement problem involving } \\
\text { arrangement and selection }\end{array}$ & 28 & 14 & 12 & 6 & 3.07 & 1.04 & $\mathrm{D}$ \\
\hline 29. & Variance & 32 & 10 & 17 & 1 & 3.22 & 0.92 & $\mathrm{D}$ \\
\hline 30. & $\begin{array}{c}\text { Coefficient of variance of binomial } \\
\text { distributions }\end{array}$ & 35 & 9 & 13 & 3 & 3.27 & 0.97 & $\mathrm{D}$ \\
\hline 31. & Coefficient of variance of Poisson distribution & 36 & 6 & 15 & 3 & 3.25 & 1.00 & $\mathrm{D}$ \\
\hline 32. & Coefficient of variance of normal distributions & 35 & 7 & 15 & 3 & 3.23 & 1.00 & $\mathrm{D}$ \\
\hline 33. & Binomial distribution & 25 & 18 & 16 & 1 & 3.12 & 0.87 & $\mathrm{D}$ \\
\hline 34. & Poisson distribution & 28 & 16 & 15 & 1 & 3.18 & 0.87 & $\mathrm{D}$ \\
\hline 35. & Normal distribution & 32 & 17 & 10 & 1 & 3.33 & 0.82 & $\mathrm{D}$ \\
\hline 36. & $\begin{array}{c}\text { Binomial approximations by Poisson } \\
\text { distributions }\end{array}$ & 33 & 13 & 13 & 1 & 3.30 & 0.87 & $\mathrm{D}$ \\
\hline 37. & $\begin{array}{c}\text { Normal approximations by binomial } \\
\text { distributions }\end{array}$ & 32 & 13 & 12 & 3 & 3.23 & 0.95 & $\mathrm{D}$ \\
\hline 38. & $\begin{array}{c}\text { Concept of correlations as measure of } \\
\text { relationship }\end{array}$ & 29 & 11 & 17 & 3 & 3.10 & 0.99 & $\mathrm{D}$ \\
\hline 39. & Scatter diagrams & 32 & 12 & 11 & 5 & 3.18 & 1.02 & $\mathrm{D}$ \\
\hline 40. & Rank correlation & 29 & 11 & 14 & 6 & 3.05 & 1.06 & $\mathrm{D}$ \\
\hline 41. & Tied ranks & 32 & 18 & 7 & 3 & 3.32 & 0.87 & $\mathrm{D}$ \\
\hline \multirow[t]{2}{*}{42.} & Classical & 27 & 10 & 17 & 6 & 2.97 & 1.07 & $\mathrm{D}$ \\
\hline & Grand Mean & & & & & 3.00 & 0.99 & $\mathrm{D}$ \\
\hline
\end{tabular}

Table 3: Mean and Standard Deviation (SD) on the Extent of Students' Perception of the

Statistics Content Difficulty in the FMC

Difficult (D); Easy (E), D=88\%; E=12\%

Data in table 3 showed that students perceived the Statistics contents of the FMC difficult to learn (Mean=3.00; $\mathrm{SD}=0.99$ ). Table 3 also revealed that the extent of students' perception of the Statistics content difficulty in the FMC was high $(88 \%)$.

- Research question four: What is the extent of students' perception of the Mechanics content difficulty in the FMC? 


\begin{tabular}{|c|c|c|c|c|c|c|c|c|}
\hline $\mathbf{S} / \mathbf{N}$ & Mechanics Contents & VD & $\mathbf{D}$ & $\mathbf{E}$ & $\mathbf{V E}$ & Mean & SD & Decision \\
\hline 1. & Scalars quantity & 19 & 14 & 18 & 9 & 2.72 & 1.08 & $\mathrm{D}$ \\
\hline 2. & Vectors quantity & 17 & 11 & 19 & 13 & 2.53 & 1.13 & $\mathrm{D}$ \\
\hline 3. & Zero vector & 14 & 17 & 18 & 11 & 2.57 & 1.05 & $\mathrm{D}$ \\
\hline 4. & Negative vector & 12 & 10 & 23 & 15 & 2.32 & 1.07 & E \\
\hline 5. & Vectors & 10 & 11 & 27 & 12 & 2.32 & 0.98 & $\mathrm{E}$ \\
\hline 6. & Vector addition and subtraction & 17 & 5 & 26 & 12 & 2.45 & 1.11 & $\mathrm{E}$ \\
\hline 7. & Scalar multiplication of vectors & 14 & 9 & 18 & 19 & 2.30 & 1.15 & E \\
\hline 8. & Magnitude and direction of a vector & 21 & 11 & 20 & 8 & 2.75 & 1.08 & $\mathrm{D}$ \\
\hline 9. & Unit vector & 17 & 14 & 22 & 7 & 2.68 & 1.02 & $\mathrm{D}$ \\
\hline 10. & The triangle law & 25 & 9 & 15 & 11 & 2.80 & 1.18 & $\mathrm{D}$ \\
\hline 11. & The parallelogram law & 22 & 5 & 19 & 14 & 2.58 & 1.21 & $\mathrm{D}$ \\
\hline 12. & Resolution of vectors & 22 & 8 & 18 & 12 & 2.67 & 1.17 & $\mathrm{D}$ \\
\hline 13. & Scalar (dot) product & 24 & 7 & 22 & 7 & 2.80 & 1.10 & $\mathrm{D}$ \\
\hline 14. & Application of scalar (dot) product & 30 & 10 & 13 & 7 & 3.05 & 1.10 & $\mathrm{D}$ \\
\hline 15. & Scalar product of vectors in three dimensions & 28 & 6 & 19 & 7 & 2.92 & 1.12 & $\mathrm{D}$ \\
\hline 16. & Application of scalar product & 28 & 12 & 13 & 7 & 3.02 & 1.08 & $\mathrm{D}$ \\
\hline 17. & Vector or cross product in three dimensions & 30 & 7 & 19 & 4 & 3.05 & 1.05 & $\mathrm{D}$ \\
\hline 18. & Application of cross product & 36 & 11 & 9 & 4 & 3.32 & 0.97 & $\mathrm{D}$ \\
\hline 19. & Newton's law of motion & 27 & 6 & 15 & 12 & 2.80 & 1.22 & $\mathrm{D}$ \\
\hline 20. & Motion along inclined plane & 35 & 1 & 13 & 11 & 3.00 & 1.25 & $\mathrm{D}$ \\
\hline 21. & Motion of connected particles & 30 & 7 & 15 & 8 & 2.98 & 1.14 & $\mathrm{D}$ \\
\hline 22. & Work & 14 & 9 & 29 & 8 & 2.48 & 1.00 & E \\
\hline 23. & Power & 10 & 5 & 37 & 8 & 2.28 & 0.90 & $\mathrm{E}$ \\
\hline 24. & Energy & 13 & 11 & 23 & 13 & 2.40 & 1.06 & $\mathrm{E}$ \\
\hline 25. & Impulse and momentum & 25 & 7 & 16 & 12 & 2.75 & 1.20 & $\mathrm{D}$ \\
\hline 26. & Projectiles & 25 & 7 & 13 & 15 & 2.70 & 1.25 & $\mathrm{D}$ \\
\hline 27. & Trajectory of projectiles & 30 & 11 & 6 & 13 & 2.97 & 1.22 & $\mathrm{D}$ \\
\hline 28. & Greatest height reached & 30 & 8 & 10 & 12 & 2.93 & 1.22 & $\mathrm{D}$ \\
\hline 29. & Time of flight & 27 & 11 & 10 & 12 & 2.88 & 1.19 & $\mathrm{D}$ \\
\hline 30. & Range & 15 & 9 & 22 & 14 & 2.42 & 1.11 & E \\
\hline 31. & Projection along inclined plane & 33 & 7 & 12 & 8 & 3.08 & 1.14 & $\mathrm{D}$ \\
\hline 32. & Forces in equilibrium & 33 & 7 & 13 & 7 & 3.10 & 1.12 & $\mathrm{D}$ \\
\hline 33. & $\begin{array}{c}\text { Resultant of parallel forces (in the same } \\
\text { direction and in opposite directions) acting on } \\
\text { a rigid body }\end{array}$ & 33 & 9 & 11 & 7 & 3.13 & 1.10 & $\mathrm{D}$ \\
\hline 34. & $\begin{array}{c}\text { Moment of a force ( } 2 \text { and } 3 \text { force) acting at a } \\
\text { point }\end{array}$ & 28 & 8 & 18 & 6 & 2.97 & 1.09 & $\mathrm{D}$ \\
\hline 35. & Polygon of forces & 31 & 10 & 15 & 4 & 3.13 & 1.02 & $\mathrm{D}$ \\
\hline 36. & Resolution of forces of friction & 22 & 10 & 22 & 6 & 2.80 & 1.05 & $\mathrm{D}$ \\
\hline 37. & Application of scalar (dot) product & 31 & 3 & 21 & 5 & 3.00 & 1.10 & $\mathrm{D}$ \\
\hline & Grand Mean & & & & & 2.77 & 1.11 & $\mathrm{D}$ \\
\hline
\end{tabular}

Table 4: Mean and Standard Deviation (SD) on the Extent of Students' Perception of the Mechanics Content Difficulty in the FMC

Difficult (D); Easy (E), D=78\%; E=22\%

Data in table 4 showed that students perceived the Mechanics content of the FMC difficult to learn (Mean=2.77; $\mathrm{SD}=1.11$ ). Table 4 also revealed that the extent of students' perception of the Mechanics content difficulty in the FMC was high (78\%).

- Research question five: What is the extent of students' perception of the Operations Research content difficulty in the FMC? 


\begin{tabular}{|l|c|c|c|c|c|c|c|c|}
\hline S/ N & Operations Research Contents & VD & $\mathbf{D}$ & $\mathbf{E}$ & $\mathbf{V E}$ & Mean & SD & Decision \\
\hline 1. & Definition of operations research & 36 & 6 & 13 & 5 & 3.22 & 1.06 & $\mathrm{D}$ \\
\hline 2. & History and nature operation research & 34 & 11 & 11 & 4 & 3.25 & 0.99 & $\mathrm{D}$ \\
\hline 3. & Models of operation research & 28 & 12 & 17 & 3 & 3.08 & 0.98 & $\mathrm{D}$ \\
\hline 4. & Linear programming model & 38 & 8 & 11 & 3 & 3.35 & 0.95 & $\mathrm{D}$ \\
\hline 5. & Transportation model & 43 & 7 & 7 & 3 & 3.50 & 0.89 & $\mathrm{D}$ \\
\hline 6. & Assignment models & 44 & 6 & 7 & 3 & 3.52 & 0.89 & $\mathrm{D}$ \\
\hline 7. & Practical application of the models & 40 & 13 & 3 & 4 & 3.48 & 0.87 & $\mathrm{D}$ \\
\hline 8. & Concept of inventory & 42 & 7 & 7 & 4 & 3.45 & 0.95 & $\mathrm{D}$ \\
\hline 9. & Definition of important terms in inventory & 42 & 6 & 7 & 5 & 3.42 & 1.00 & $\mathrm{D}$ \\
\hline 10. & Holding list & 35 & 4 & 14 & 7 & 3.12 & 1.14 & $\mathrm{D}$ \\
\hline 11. & Demand & 26 & 19 & 10 & 5 & 3.10 & 0.97 & $\mathrm{D}$ \\
\hline 12. & Ordering list & 37 & 6 & 12 & 5 & 3.25 & 1.05 & $\mathrm{D}$ \\
\hline 13. & Computation of optimal quantity (EOQ model) & 40 & 8 & 9 & 3 & 3.42 & 0.93 & $\mathrm{D}$ \\
\hline 14. & Concept of replacement & 34 & 13 & 10 & 3 & 3.30 & 0.93 & $\mathrm{D}$ \\
\hline 15. & Individual replacement sudden failure item & 45 & 4 & 8 & 3 & 3.52 & 0.91 & $\mathrm{D}$ \\
\hline 16. & Replacement of items that wear out gradually & 37 & 9 & 10 & 4 & 3.32 & 0.98 & $\mathrm{D}$ \\
\hline 17. & Introduction of modeling & 44 & 4 & 8 & 4 & 3.47 & 0.96 & $\mathrm{D}$ \\
\hline 18. & Dependent and independent variables in & 39 & 10 & 6 & 5 & 3.38 & 0.98 & $\mathrm{D}$ \\
& mathematical modeling & & & & & & & \\
\hline 19. & Examples of some models & 33 & 8 & 11 & 8 & 3.10 & 1.13 & $\mathrm{D}$ \\
\hline 20. & Construction of model & 31 & 13 & 8 & 8 & 3.12 & 1.09 & $\mathrm{D}$ \\
\hline 21. & Methodology of modeling & 38 & 10 & 4 & 8 & 3.30 & 1.08 & $\mathrm{D}$ \\
\hline 22. & Application to physical, biological, social and & 31 & 11 & 7 & 11 & 3.03 & 1.18 & $\mathrm{D}$ \\
\hline 23. & behavioural services. & & & & & & & \\
\hline 24. & Introduction to game theory. & 32 & 10 & 7 & 11 & 3.05 & 1.19 & $\mathrm{D}$ \\
\hline 25. & Description of types of games. & 31 & 13 & 7 & 9 & 3.10 & 1.12 & $\mathrm{D}$ \\
\hline 26. & Solution of two persons zero sum games using & 34 & 8 & 7 & 11 & 3.08 & 1.20 & $\mathrm{D}$ \\
\hline & pure and mixed strategies. & & & & & & & \\
\hline & Matrix games. & & & & 31.27 & 1.02 & $\mathrm{D}$ \\
\hline
\end{tabular}

Table 5: Mean and Standard Deviation (SD) on the Extent of Students Perception of the Operations Research Content Difficulty in the FMC Difficult (D); Easy (E), D=100\%; E=0\%

Data in table 5 showed that students perceived the Operations Research content of the FMC difficult to learn $($ Mean $=3.27 ; \mathrm{SD}=1.02)$. Table 5 also revealed that the extent of students' perception of the operations research content difficulty in the FMC was high (100\%).

- Research question six: What is the extent of students' perception of the FMC Themes difficulty?

\begin{tabular}{|c|c|c|c|c|c|c|}
\hline S/ N & FMC Themes & $\begin{array}{c}\text { No of } \\
\text { Contents }\end{array}$ & Mean & SD & Difficult (\%) & Easy (\%) ) \\
\hline 1 & Pure Mathematics & 140 & 2.82 & 0.98 & $105(75 \%)$ & $35(25 \%)$ \\
\hline 2 & Coordinate Geometry & 18 & 3.08 & 0.99 & $18(100 \%)$ & $0(0 \%)$ \\
\hline 3 & Statistics & 42 & 3.00 & 0.99 & $37(88 \%)$ & $5(12 \%)$ \\
\hline 4 & Mechanics & 37 & 2.77 & 1.11 & $29(78 \%)$ & $8(22 \%)$ \\
\hline 5 & Operations Research & 26 & 3.27 & 1.02 & $26(100 \%)$ & $0(0 \%)$ \\
\hline Total & 263 & 2.99 & 1.02 & \multicolumn{2}{|c|}{$215(88.20 \%)$} & $48(11.80 \%)$ \\
\hline
\end{tabular}

Table 6: Mean, Standard Deviation (SD) and Simple Percentage on the Extent of Students' Perception of the FMC Content Difficulty

Data in table 6 showed that students perceived the Further Mathematics curriculum themes difficult to learn (Mean=2.99; $\mathrm{SD}=1.02$ ). Table 6 also revealed that the extent of students' perception of the Further Mathematics curriculum themes difficulty was high $(88.20 \%)$.

\section{Discussion of Findings}

4.1. Assessment of the Extent of Student Perception of the Pure Mathematics Content Difficulty in the FMC

Data in table 1 revealed that students perceived the pure mathematics content of the FMC difficult to learn $(\mathrm{M}=2.82 ; \mathrm{SD}=0.98)$. Table 1 also showed that the extent of student perception of the pure mathematics content difficulty in the FMC was high (75\%). An analysis of topics perceived difficult by Nigerian students and teachers in secondary school 
Further Mathematics curriculum by Ifamuyiwa (2014) revealed that some topics in pure mathematics such as differential (differentiation) and integral (integration) calculus were difficult with students to learn.

\subsection{Determination of the Extent of Student Perception of the Coordinate Geometry Content Difficulty in the Fmc}

Data in table 2 revealed that students perceived the coordinate geometry content of the FMC difficult to learn $(\mathrm{M}=3.08 ; \mathrm{SD}=0.99)$. Table 2 also showed that the extent of student perception of the coordinate geometry content difficulty in the FMC was high (100\%). Wonu and Zalmon (2017) diagnosed coordinate geometry as one of students 'common learning difficulties in geometry. Zalmon and George (2018) reported that students perceived geometry and introductory calculus themes difficult to learn. Further study by Iji and Omenka (2015) identifiedthe contents in the coordinate geometry theme that students perceived difficult to learn as equation of parabola in rectangular Cartesian coordinate and equation of ellipse in rectangular Cartesian coordinate.

\subsection{Ascertainment of the Extent of Student Perception of the Statistics Content Difficulty in the FMC}

Data in table 3 showed that students perceived the statistics content of the FMC difficult to learn (M=3.00; $\mathrm{SD}=0.99$ ). Table 3 also revealed that the extent of student perception of the statistics content difficulty in the FMC was high (88\%). Wonu and Zalmon (2017) noted earlier that students have difficulties in learning to use the histogram to estimate the mode, differentiate between bar chart and histogram, estimate quartiles and percentiles, using ogive, calculating standard deviation and representing information in a diagram.

\subsection{The Extent of Student Perception of the Mechanics Content Difficulty in the FMC}

Data in table 4 showed that students perceived the mechanics content of the FMC difficult to learn $(M=2.77$; $\mathrm{SD}=1.11$ ). Table 4 also revealed that the extent of student perception of the mechanics content difficulty in the FMC was high (78\%).Iji and Omenka (2015) identified the contents in the mechanics theme that students perceived difficult to learn as motion of connected particles, polygon of forces, trajectory of projectile and moment of force ( 2 and 3 force) acting at a point.

\subsection{Students' Perception of the Operations Research Content Difficulty in the FMC}

Data in table 5 showed that students perceived the operations research content of the FMC difficult to learn $(\mathrm{M}=3.27 ; \mathrm{SD}=1.02)$. Table 5 also revealed that the extent of student perception of the operations research content difficulty in the FMC was high (100\%). An analysis of topics perceived difficult by Nigerian students and teachers in secondary school Further Mathematics curriculum by Ifamuyiwa (2014) also revealed that the operations research theme was difficult with students to learn. Iji and Omenka (2015) revealed that the perceived learning difficulty in operations research was due to lack of adequate instructional materials.

\subsection{Assessing the Extent of Student Perception of the FMC Content Difficulty}

Data in table 6 showed that students perceived the Further Mathematics curriculum content difficult to learn $(\mathrm{M}=2.99 ; \mathrm{SD}=1.02)$. Table 6 also revealed that the extent of student perception of the Further Mathematics curriculum content difficulty was high (88.20\%). Earlier study by Mills (2011) revealed that more than $30 \%$ of students in schools today have significant difficulties in learning Further Mathematics in spite of normal intelligence. Mills (2011) attributed this learning difficulty mainly to students' inadequate preparation and ineffective early education in the underlying basic mathematical operations that are required for Mathematics studies at their current level. Iji and Omenka (2015) identified the Further Mathematics contents perceived difficult with students as coordinate geometry, mechanics and operations research.

\section{Conclusion}

The study concluded that students' perception of content difficulty in the Further Mathematics curriculum was high (88.20\%) and that the difficulty was in all the FMC themes of Pure Mathematics (75\%), Coordinate Geometry (100\%), Statistics (88\%), Mechanics (78\%) and Operations Research (100\%).

\section{Recommendations}

Based on the findings the study recommended as follows:

- The Nigerian Educational Research and Development Council (NERDC) should carry out a holistic review of the Further Mathematics curriculum in view of students' high perception of its content difficulty.

- Public school proprietors and educational administrators should ensure instructional effectiveness through adequate provision of learning resources including qualified Further Mathematics teachers and training and retraining of in-service Further Mathematics teachers through sponsorship to attend workshops, seminars, symposia and conferences.

- Students should be encouraged to develop positive attitudes towards learning Further Mathematics through diligence and improved study habit.

\section{References}

i. Charles-Ogan, G.I. \& George, N.R. (2019). Further mathematics content coverage and first year students' algebra and calculus performance in universities in Rivers State Nigeria. American Journal of Mathematics and Statistics, 9(3), 115-122. 
ii. Federal Republic of Nigeria (2014). National policy on education (4th ed.). Lagos: Nigerian Educational Research and Development Council (NERC) Press.

iii. Ifamuyiwa, A.S. (2014). Analysis of topics perceived difficult by Nigerian students and teachers in secondary school further mathematics. Abacus: Journal of Mathematical Association of Nigeria, 39(1), 255-268.

iv. Iji, C. O. \& Omenka, J. E. (2015). Mathematics teachers' perception of difficult concepts in secondary school Mathematics curriculum in Benue State, Nigeria. Asia Pacific Journal of Education, Arts and Sciences, 2(1), 32 - 45.

v. Mills, D. (2011). Mathematics learning difficulties. Retrieved May 15, 2019 from http:/ / www.mathlearningdifficulties.com

vi. Wonu N. \& Zalmon I.G. (2017). Diagnosis and remediation of senior secondary student common learning difficulties in Mathematics from chief examiners' report. European Journal of Research and Reflection in Educational Sciences,5(1), 256-282.

vii. Zalmon, I.G. \& George, N.R. (2018). Student and teacher perception of content difficulty in the Nigerian senior secondary Mathematics curriculum, International Journal of Mathematics Trends and Technology (IJMTT), 63(3), 157-168.

viii. Zalmon, I.G. \&Wonu, N. (2017). Comparative analysis of student Mathematics achievement in West African senior secondary certificate examination in Nigeria. European Journal of Research and Reflection in Educational Sciences, $5(1), 24-31$. 